\title{
SHM and Efficient Strategies for Reduced-Order Modeling ${ }^{\dagger}$
}

\author{
Giorgio Gobat ${ }^{1}$, Saeed Eftekhar Azam ${ }^{1,2}$ and Stefano Mariani ${ }^{1, *}$ (1) \\ 1 Department of Civil and Environmental Engineering, Politecnico di Milano, Piazza L. da Vinci 32, \\ 20133 Milano, Italy; giorgio.gobat@polimi.it (G.G.); Saeed.EftekharAzam@unh.edu (S.E.A.) \\ 2 Department of Civil and Environmental Engineering, University of New Hampshire, 33 Academic Way, \\ W 137, Durham, NH 03824, USA \\ * Correspondence: stefano.mariani@polimi.it; Tel.: +39-02-2399-4279 \\ + Presented at the 7th Electronic Conference on Sensors and Applications, 15-30 November 2020; \\ Available online: https:/ / ecsa-7.sciforum.net/.
}

Published: 2 March 2021

check for

\begin{abstract}
Within model-based approaches to structural health monitoring (SHM), numerical simulations must be tailored to continuously adapt to the degradation processes and to the possibly changing environment. This model update stage of the analysis brings two competing requirements: the accuracy of the model, with a more detailed description of the phenomena required where damage is supposed to take place; the efficiency of the model, to reduce the overall computational burden and allow for real-time (or close to real-time) computing. Without resorting to AI-based strategies, approaches solely based on proper orthogonal decomposition (POD) and domain decomposition (DD) techniques proved rather efficient in handling the aforementioned trade-off between the diverging requirements of accuracy and efficiency. In this work, we discuss a further improvement over our recently proposed methodology that consists of: a DD of the entire structure into sub-regions, which can be designed to decouple regions more prone to get damaged from regions that are instead less affected by the degradation processes; a POD-based selective model order reduction for all the domains, with adjustable and heterogeneous accuracy requirements. The approach is assessed through an illustrative example related to beam dynamics, with results provided in terms of both accuracy and computational efficiency, or speedup with respect to the full-order model.
\end{abstract}

Keywords: structural health monitoring; numerical simulations; reduced-order modeling; proper orthogonal decomposition; domain decomposition

\section{Introduction}

Civil structures and infrastructure systems are nowadays subjected to aging and increased extreme loadings, sometimes caused by the climate change [1]. The latter can lead to a structural damage, that is a reduction of the structural stiffness and strength or load bearing capacity [2]. Since a strength reduction cannot be directly measured at the structural level, and can often be overlooked at its early stages, indirect ways must be envisioned to infer its effects from the structural output. Structural health monitoring (SHM) aims at processing the information brought by measurements collected e.g., through a network of sensors deployed over the structure, compared with the output of a reference damage-free configuration and estimating the damage pattern. This procedure is typically referred to as model-based whenever the aforementioned damage-free solution is provided by a numerical (e.g., finite element, FE) model of the structure in its virgin state, possibly tuned during an initial update/training stage to account for the relevant epistemic uncertainties. An alternative approach, avoiding any comparison of the collected data with physics-based models of the structural system, 
is referred to as data-driven [3]: for instance, unsupervised classification of the current state and early damage detection may consist of learning the output of the undamaged structure, even under environmental and load variabilities, and quantifying a drift from the reference baseline. Machine learning and deep learning solutions are currently investigated [4-7], to enhance this approach and attain the same level of accuracy of model-based solutions.

In this work, we focus on a model-based framework for SHM and propose a robust and efficient way to reduce the computational burden of the numerical models, whose output must be compared to the noisy experimental data. Computational efficiency is of paramount importance for the comparison stage, to perform diagnosis and prognosis of damage in a timely fashion. In fact, in our smart cities age, the damage forecast has to be provided in real-time; on the fly while measurements are collected, to immediately process them and warn in case of a degradation of the structural health. Accordingly, real-time (big) data analytics can exploit Kalman or particle filtering for the relevant estimation of damage [8-13].

To speed up the simulations, proper orthogonal decomposition (POD) proved efficient thanks to its inherent capability to furnish a priori estimates of the overall accuracy of the reduced-order model (ROM) that it delivers; see $[14,15]$. POD is ruled, like the mentioned machine and deep learning strategies, by a training stage that aims at digging into the data provided by the original numerical model and/or by the acquired measurements, to establish patterns or structures in them, that have to be exploited next to enhance the model efficiency. Even if it works in a way similar to an eigenvalue problem to set the structural vibration modes, it does not require a priori knowledge of the mass and stiffness properties of the entire structure; the other way around, as training is conducted under specific loading conditions, the ROM capacity to mimic the reality is assured only under the same conditions and might get largely spoiled under environmental variability. To further improve its computational gain, in [16], we already proposed a joint use of POD and demain decomposition (DD); see also [17-19]: different system components can be accordingly handled with heterogeneous accuracy measures, depending on the relevance they have at the structural level.

Even though the proposed joint implementation of POD and DD by the authors [16] was a promising tool for real-time simulations, it did not prove easy and optimal to move towards real-time damage estimation. To address those shortcomings, here we discuss an alternative procedure for its implementation. This alternative procedure is shown to have advantages in terms of the simulation accuracy, which is obtained via enforcing certain conditions to be satisfied at the interfaces between different sub-domains (SDs) of the DD method. In addition, in the interest of implementation simplicity, the computations are carried out in an iterative fashion, which needless to say, leads to a reduction of the computational efficiency, that must be accounted for as well at the strategy optimization level, see also $[20,21]$.

\section{Methodology}

Upon space discretization, the equation governing structural dynamics turns out to be:

$$
\mathbf{M} \ddot{\mathbf{u}}+\mathbf{F}^{\text {int }}(\mathbf{u})=\mathbf{F}^{\text {ext }}
$$

where, in the considered undamped case: $\mathbf{M}$ is the mass matrix; $\mathbf{u}$ and $\ddot{\mathbf{u}}$ are the vectors of nodal displacements and accelerations; $\mathbf{F}^{\text {int }}$ is the vector of internal forces linked to the (possibly) nonlinear material behavior; $\mathbf{F}^{\text {ext }}$ is the vector of external forces.

By subdividing the entire structure into $N$ non-overlapping SDs, for each one of them, the solution is enforced in the linear-elastic case given by $\mathbf{F}^{\text {int }}(\mathbf{u})=\mathbf{K u}, \mathbf{K}$ being the stiffness matrix of the structure, through:

$$
\mathbf{M}_{s} \ddot{\mathbf{u}}_{s}+\mathbf{K}_{s} \mathbf{u}_{s}=\mathbf{F}_{s}^{\text {ext }}+\mathbf{C}_{s}^{T} \boldsymbol{\Lambda}, \quad s=1, \ldots, N
$$

where, besides the same entries of Equation (1) with the subscript $s$ to denote that the solution refers to the $s$-th SD only, the second term at the right hand side represents the interaction of the considered SD 
with the surrounding ones. In this latter term, $\mathrm{C}_{s}$ is a Boolean matrix, while $\Lambda$ is a vector of interface forces representing the effects of the tractions taking all the numerical SDs joined together.

The solution to Equation (2) is sought in terms of displacement components as:

$$
\mathbf{u}_{s}=\mathbf{u}_{s}^{\text {free }}+\mathbf{u}_{s}^{\text {link }}
$$

namely by decomposing the response into the components relevant to the external actions (represented by $\mathbf{u}_{s}^{\text {free }}$ ) and to the interface forces (represented by $\mathbf{u}_{s}^{\text {link }}$ ). While the contribution $\mathbf{u}_{s}^{\text {free }}$ can be rather easily computed once rigid body modes are filtered out, the term $\mathbf{u}_{s}^{\text {link }}$ largely depends on the strategy adopted to set $\Lambda$. For the sake of brevity, the details of the procedure are omitted; it is noteworthy that the stability and accuracy issues evidenced in [16] were due to the adopted non-iterative DD strategy, as proposed in [22] to compute $\mathbf{u}_{s}^{\text {link }}$. To improve those results, we rely here upon the iterative strategy proposed instead in [23].

The first computational gain provided by DD basically stems from the possibility to solve for $\mathbf{u}_{s}^{\text {free }}$ and $\mathbf{u}_{s}^{\text {link }}$ using a parallel code implementation that in theory would allow, for everything but the setting of vector $\Lambda$, to scale the computing time as $1 / N$. The second computational gain here explored rests on POD, see $[14,15,24]$ for details: once the solution $\mathbf{u}_{s}$ for each SD has been computed for a set of time instants, a matrix of so-called snapshots can be assembled and decomposed to compute the relevant proper orthogonal modes (POMs). Finally, by retaining in the ROM the minimum sufficient number of POMs with an oriented energy content high enough to assure (energetic) surrogate model accuracy, the ROM itself can be obtained by a Galerkin projection of Equation (2) onto the space spanned by the mentioned POMs. For SHM purposes, this last step typically allows reducing the order of the problem to solve by orders of magnitude, as testified by the results related to the example to follow.

\section{Results}

To start assessing the capability of the proposed approach, the cantilever beam depicted in Figure 1 has been considered. The beam is assumed to be clamped on its left side (at $x=0$ ), and loaded along its right free edge (at $x=4 \mathrm{~m}$ ) by a vertical load $F=40 \mathrm{kN}$ applied at time $t=0$. The beam is initially at rest in an unstressed and undamaged state, and made of steel. It has been space-discretized as shown in the figure, with a mesh consisting of 560 two-dimensional constant-strain triangular elements and 340 nodes; allowing for the constraints along its left side, the total number of degrees-of-freedom (DOFs) in the full-order model (FOM) has turned out to be 664. As can be also seen in the figure, the beam has been meshed so as to allow managing up to $N=4$ SDs in the analyses.

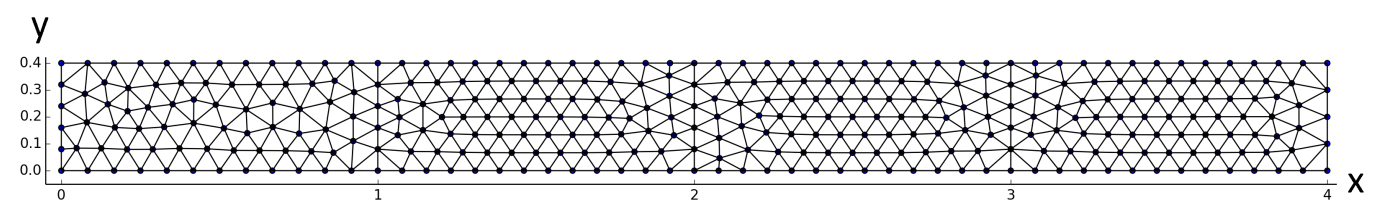

Figure 1. Cantilever geometry, reference system and adopted finite element (FE) mesh.

The simulated structural response is reported in Figure 2 in terms of time histories of the vertical displacement and velocity of the nodes along the free edge, where the load has been applied. Results are compared among: the FOM solution, without any model order reduction (solution denoted as full in the figure legend); the solution obtained by means of POD only, with no DD (mono in the legend); the solutions obtained through the joint use of POD and DD, handling $N=2,3$ or 4 SDs (respectively denoted as 2SD, 3SD and 4SD in the legend). The training time has been set to $0.02 \mathrm{~s}$ for all the simulations; this is the reason why the ROM solutions look smoother than the FOM one starting at $t=0.02 \mathrm{~s}$, and they also show a slightly different frequency content testified by the drift in the oscillations. These effects are obviously more evident in the velocity time history, and allow 
assessing the procedure property of filtering high-frequency oscillations not retained in the ROMs, independently of the number of adopted SDs.

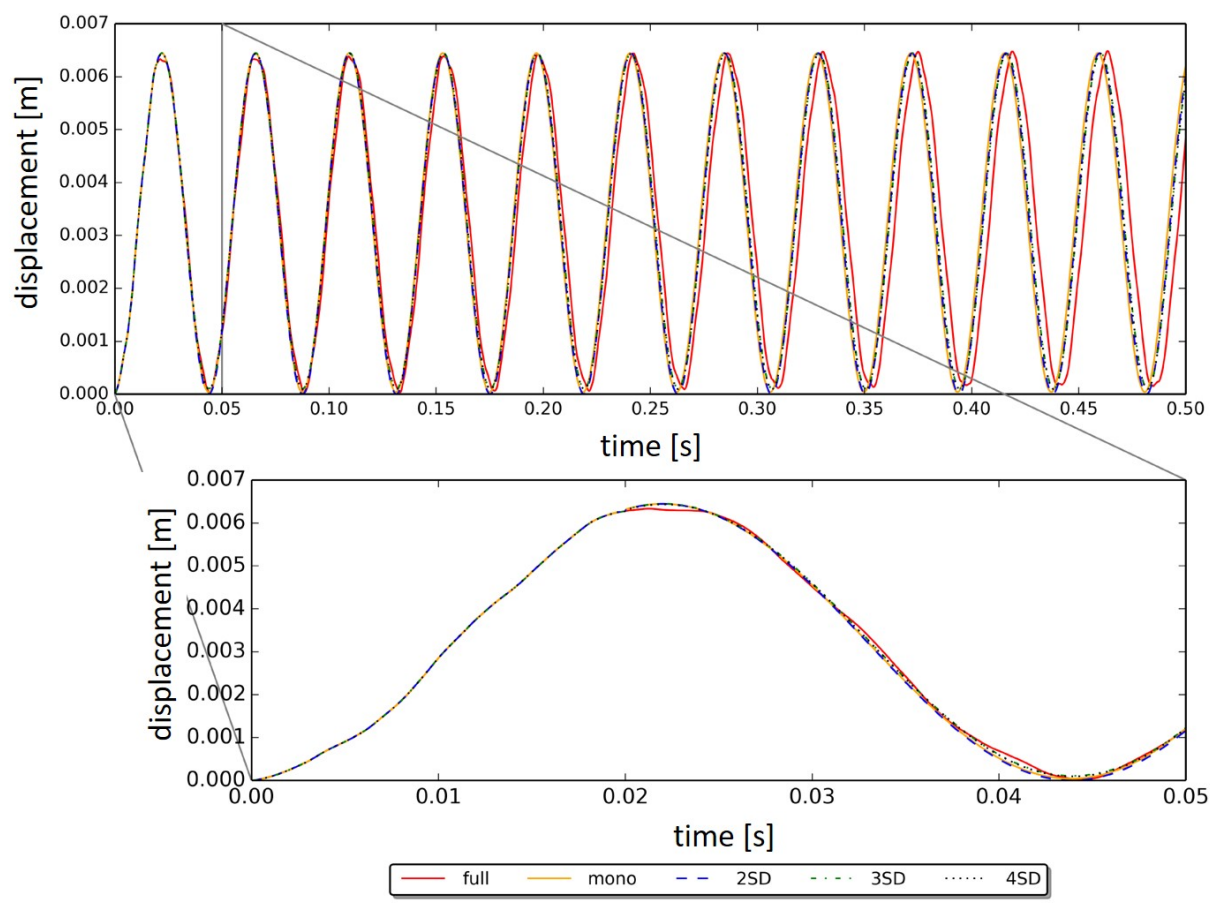

(a)
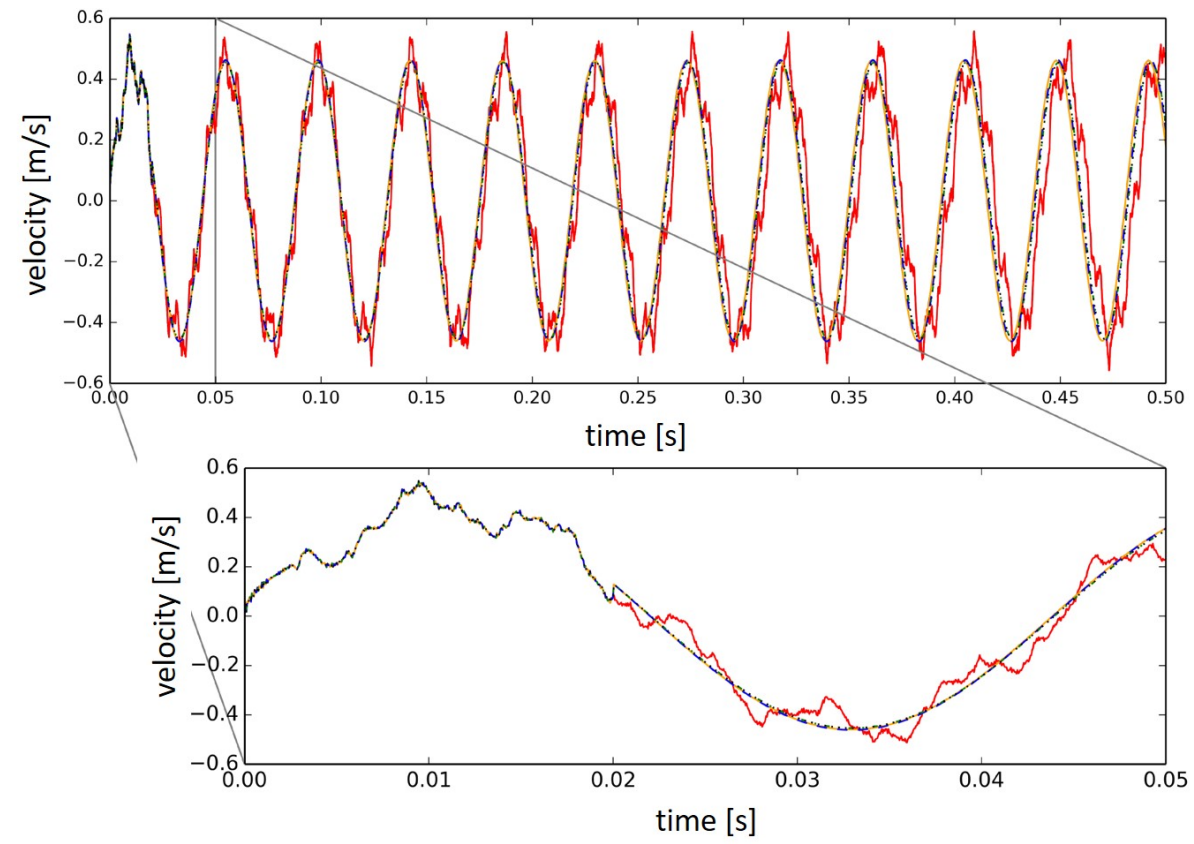

(b)

Figure 2. Effects of the number of the adopted sub-domains (SDs) on the time evolutions of vertical tip (a) displacement and (b) velocity.

The attained speedup values are all reported in Figure 3 and Table 1. The results, represented in each case by the ratio between the CPU times required by the FOM analysis and by the ROM one, are here compared not only at varying number $N$ of the adopted SDs, but also at varying time step size, to assure results accuracy. To attain an energy accuracy of $99 \%$, see e.g., $[16,24,25]$, it is reported 
that one POM only has to be retained in the ROM for each SD. This strong reduction in the number of the DOFs from the original 664 to 1 per SD, leads to the reported values of the speedup of up to 280. It is also shown that, for each value of the time step size, the speedup does not decrease as $1 / N$, but much faster: this issue is linked to the fact that only the free problem of DD has been reduced in its order, while the link one dealing with the interactions between SDs has not. Furthermore, to attain a comparable speedup in all the analyses, or avoid at least the mentioned $1 / N$ trend, parallel computing should be exploited.

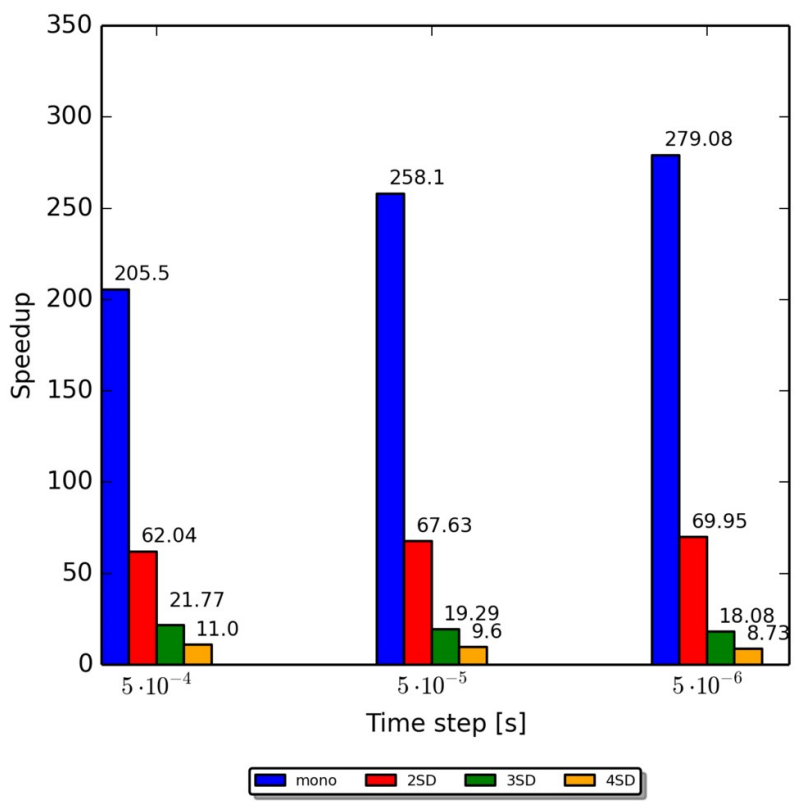

Figure 3. Effects of the number of the adopted SDs on the attained speedup, at varying time step size.

Table 1. Details on the effects of the number of the adopted SDs on the elapsed time of each analysis and on the attained speedup, at varying time step size.

\begin{tabular}{ccccc}
\hline SDs & Time Step [s] & POMs & Elapsed Time [s] & Speedup \\
\hline \multirow{3}{*}{1} & $5 \times 10^{-4}$ & 1 & 0.35 & 205.5 \\
& $5 \times 10^{-5}$ & 1 & 1.95 & 258.1 \\
& $5 \times 10^{-6}$ & 1 & 18.1 & 279.08 \\
\hline \multirow{3}{*}{2} & $5 \times 10^{-4}$ & 1 & 0.81 & 62.04 \\
& $5 \times 10^{-5}$ & 1 & 7.34 & 67.63 \\
& $5 \times 10^{-6}$ & 1 & 7.2 & 69.95 \\
\hline \multirow{3}{*}{3} & $5 \times 10^{-4}$ & 1 & 2.35 & 21.77 \\
& $5 \times 10^{-5}$ & 1 & 26.3 & 19.29 \\
& $5 \times 10^{-6}$ & 1 & 280 & 18.08 \\
\hline \multirow{3}{*}{4} & $5 \times 10^{-4}$ & 1 & 4.6 & 11 \\
& $5 \times 10^{-5}$ & 1 & 52.8 & 9.6 \\
& $5 \times 10^{-6}$ & 1 & 580 & 8.73 \\
\hline
\end{tabular}

The most interesting result to notice in Table 1 is represented by the elapsed time relevant to the analysis featuring a time step size of $5 \times 10^{-4} \mathrm{~s}$ (the largest here adopted) and no DD: for an analysis of the structural response till $0.5 \mathrm{~s}$, the sequential simulation has lasted $0.35 \mathrm{~s}$ with a computer featuring a rather cheap Intel I7-4500 U $1.8 \mathrm{GHz}$ processor. Such outcome testifies that the offered reduced-order methodology allows the simulations to run faster than the real-time, so that data processing and damage identification can be foreseen to carry out online. 


\section{Conclusions}

In this paper, we have discussed a reduced order model-based approach to SHM that allows attaining accuracy and avoids some of the drawbacks highlighted in our previous investigations. In compliance with a route to real-time monitoring, the numerical model of the structural systems was reduced in its order with an innovative, joint use of DD and POD. The results of a simple benchmark problem, consisting of a cantilever beam dynamically excited by a tip load, have shown that the approach can be adopted even for structures featuring complexity in their geometry and mechanical behavior, and can therefore allow foreseeing real-time SHM strategies.

In future works, the proposed approach will be investigated in order to highlight possible bottlenecks relevant to practical applications with real life structural systems. By coupling the proposed procedure with on-the-fly model update tools whenever damage is incepted and detected in the structure, see e.g., [26-28], the entire procedure is going to be tested for long-term SHM situations. The usage of hybrid model-based and data-driven solutions $[7,29]$ is currently and will be further investigated, with the aim of exploiting the strengths of each approach and then enhance computational efficiency and damage detectability.

Author Contributions: Conceptualization, data curation, methodology and supervision, G.G. and S.M.; software, G.G.; formal analysis, investigation, validation and writing, all. All authors have read and agreed to the published version of the manuscript.

Funding: This research received no external funding.

Acknowledgments: S.E.A. wishes to acknowledge the financial support from Politecnico di Milano for Senior Residents.

Conflicts of Interest: The authors declare no conflict of interest.

\section{References}

1. Ayyub, B.M. (Ed.) Climate-Resilient Infrastructure: Adaptive Design and Risk Management; American Society of Civil Engineers: 2018.

2. Achenbach, J.D. Structural health monitoring-What is the prescription? Mech. Res. Commun. 2009, $36,137-142$.

3. Farrar, C.R.; Worden, K. Structural Health Monitoring: A Machine Learning Perspective; John Wiley \& Sons: 2012.

4. Entezami, A.; Shariatmadar, H.; Mariani, S. Fast unsupervised learning methods for structural health monitoring with large vibration data from dense sensor networks. Struct. Health Monit. 2020, 19, 1685-1710.

5. Entezami, A.; Shariatmadar, H.; Mariani, S. Early damage assessment in large-scale structures by innovative statistical pattern recognition methods based on time series modeling and novelty detection. Adv. Eng. Softw. 2020, 150, 102923.

6. Entezami, A.; Sarmadi, H.; Behkamal, B.; Mariani, S. Big Data analytics and structural health monitoring: A statistical pattern recognition-based approach. Sensors 2020, 20, 2328.

7. Rosafalco, L.; Manzoni, A.; Mariani, S.; Corigliano, A. Fully convolutional networks for structural health monitoring through multivariate time series classification. Adv. Model. Simul. Eng. Sci. 2020, 7, 38.

8. Corigliano, A.; Mariani, S. Identification of a constitutive model for the simulation of time-dependent interlaminar debonding processes in composites. Comput. Methods Appl. Mech. Eng. 2002, 191, 1861-1894.

9. Corigliano, A.; Mariani, S. Parameter identification in explicit structural dynamics: performance of the extended Kalman filter. Comput. Methods Appl. Mech. Eng. 2004, 193, 3807-3835.

10. Mariani, S.; Corigliano, A. Impact induced composite delamination: state and parameter identification via joint and dual extended Kalman filters. Comput. Methods Appl. Mech. Eng. 2005, 194, 5242-5272.

11. Eftekhar Azam, S.; Ghisi, A.; Mariani, S. Parallelized sigma-point Kalman filtering for structural dynamics. Comput. Struct. 2012, 92-93, 193-205.

12. Chatzi, E.; Smyth, A. Particle filter scheme with mutation for the estimation of time-invariant parameters in structural health monitoring applications. Struct. Control Health Monit. 2013, 20, 1081-1095. 
13. Mariani, S.; Gobat, G. Identification of strength and toughness of quasi-brittle materials from spall tests: A Sigma-point Kalman filter approach. Inverse Probl. Sci. Eng. 2019, 27, 1318-1346.

14. Kerschen, G.; Golinval, J. Physical Interpretation of the Proper Orthogonal Modes Using the Singular Value Decomposition. J. Sound Vib. 2002, 249, 849-865.

15. Kerschen, G.; Golinval, J.C.; Vakakis, A.F.; Bergman, L.A. The Method of Proper Orthogonal Decomposition for Dynamical Characterization and Order Reduction of Mechanical Systems: An Overview. Nonlinear Dyn. 2005, 41, 147-169.

16. Corigliano, A.; Dossi, M.; Mariani, S. Model order reduction and domain decomposition strategies for the solution of the dynamic elasto-plastic structural problem. Comput. Methods Appl. Mech. Eng. 2015, 290, 127-155.

17. Faucher, V.; Combescure, A. Local modal reduction in explicit dynamics with domain decomposition. Part 2: Specific interface treatment when modal subdomains are involved. Int. J. Numer. Methods Eng. 2004, 61, 69-95.

18. Corigliano, A.; Dossi, M.; Mariani, S. Domain decomposition and model order reduction methods applied to the simulation of multiphysics problems in MEMS. Comput. Struct. 2013, 122, 113-127.

19. Corigliano, A.; Confalonieri, F.; Dossi, M.; Mariani, S. Coupled domain decomposition-proper orthogonal decomposition methods for the simulation of quasi-brittle fracture processes. Adv. Model. Simul. Eng. Sci. 2016, 3, 28.

20. Capellari, G.; Chatzi, E.; Mariani, S. Cost-benefit optimization of structural health monitoring sensor networks. Sensors 2018, 18, 2174.

21. Capellari, G.; Chatzi, E.; Mariani, S. Structural Health Monitoring Sensor Network Optimization through Bayesian Experimental Design. ASCE-ASME J. Risk Uncertain. Eng. Syst. Part A Civ. Eng. 2018, 4, 04018016.

22. Gravouil, A.; Combescure, A. Multi-time-step explicit-implicit method for non-linear structural dynamics. Int. J. Numer. Methods Eng. 2001, 50, 199-225.

23. Mahjoubi, N.; Gravouil, A.; Combescure, A.; Greffet, N. A monolithic energy conserving method to couple heterogeneous time integrators with incompatible time steps in structural dynamics. Comput. Methods Appl. Mech. Eng. 2011, 200, 1069-1086.

24. Eftekhar Azam, S.; Mariani, S. Investigation of computational and accuracy issues in POD-based reduced order modeling of dynamic structural systems. Eng. Struct. 2013, 54, 150-167.

25. Eftekhar Azam, S. Online Damage Detection in Structural Systems; Springer: 2014.

26. Capellari, G.; Eftekhar Azam, S.; Mariani, S. Damage detection in flexible plates through reduced-order modeling and hybrid particle-Kalman filtering. Sensors 2016, 16, 2.

27. Eftekhar Azam, S.; Mariani, S.; Attari, N. Online damage detection via a synergy of proper orthogonal decomposition and recursive Bayesian filters. Nonlinear Dyn. 2017, 89, 1489-1511.

28. Eftekhar Azam, S.; Mariani, S. Online damage detection in structural systems via dynamic inverse analysis: A recursive Bayesian approach. Eng. Struct. 2018, 159, $28-45$.

29. Rosafalco, L.; Torzoni, M.; Manzoni, A.; Mariani, S.; Corigliano, A. Online structural health monitoring by model order reduction and deep learning algorithms. 2020. Submitted.

Publisher's Note: MDPI stays neutral with regard to jurisdictional claims in published maps and institutional affiliations.

(C) 2021 by the authors. Licensee MDPI, Basel, Switzerland. This article is an open access article distributed under the terms and conditions of the Creative Commons Attribution (CC BY) license (http:/ / creativecommons.org/licenses/by/4.0/). 